\title{
Efectos de la conducción eficiente en el tráfico y las emisiones mediante micro-simulación
}

\author{
Álvaro García-Castro \\ Investigador, TRANSyT-UPM, España \\ Andrés Monzón de Cáceres \\ Catedrático, Universidad Politécnica de Madrid, España
}

\section{RESUMEN}

El transporte por carretera es uno de los principales causantes de la emisión de gases de efecto invernadero y del deterioro de la calidad del aire en nuestras ciudades. Para atajar este problema las administraciones competentes están promoviendo diferentes medidas entre las que se encuentra el fomento de una conducción eficiente.

La conducción eficiente comprende variadas recomendaciones para conseguir disminuir el consumo de combustible y las emisiones, entre las que se pueden citar conducir con marchas altas, evitar aceleraciones y frenadas bruscas o el mantenimiento de una distancia de seguridad suficiente para reaccionar suavemente a las variaciones del tráfico.

Muchos estudios cifran los ahorros de combustible individuales entre el 5\% y el 20\%, pero apenas se encuentran investigaciones acerca de resultados globales según se incorporan más conductores eficientes al corredor o zona de estudio.

En esta ponencia se describe el proceso de simulación de conducción eficiente para diferentes ratios de penetración entre los conductores. Para ello se parte de un análisis estadístico de perfiles de velocidad reales recogidos en ciertos itinerarios de Madrid, realizados primero con una conducción normal y posteriormente siguiendo las recomendaciones de un curso de conducción eficiente. A partir de este análisis se calibra el modelo de micro-simulación (Vissim), para posteriormente plantear diversos escenarios en función de la intensidad de tráfico y del porcentaje de conductores eficientes y calcular las emisiones mediante el modelo VERSIT+. Entre los problemas medioambientales más trascendentales para la sociedad, se encuentra el del cambio climático así como el de la calidad del aire en nuestras áreas metropolitanas.

Los resultados muestran que los ahorros individuales son mayores en porcentaje que los ahorros globales. Incluso, en el escenario con mayor congestión, un mayor porcentaje de conductores eficiente provoca una mayor congestión y por tanto un aumento de las emisiones de $\mathrm{CO}_{2}$ у $\mathrm{NO}_{x}$.

\section{INTRODUCCIÓN}

\subsection{Emisiones del sector del transporte por carretera}

El transporte por carretera es uno de los principales causantes de emisiones de gases de efecto invernadero (GEI) (Ramanathan y Feng, 2008), aportando alrededor del 20\% del 
total en Europa (European Environment Agency, 2013).

Además del problema de emisiones de GEI, otro asunto de gran interés es la calidad del aire en zonas urbanas. Según la European Environment Agency (2010), durante el periodo 1997-2008, un 62\% de la población europea que vive en áreas urbanas ha estado expuesto a valores de partículas, ozono o dióxido de nitrógeno por encima de los límites.

Las administraciones públicas se enfrentan a estos problemas desde varios ángulos: Cambios a modos de transporte más limpios, nuevas tecnologías y combustibles en los vehículos, gestión de la demanda y el uso de tecnologías de la información y la comunicación (ICT) aplicadas al transporte. Entre estas medidas destaca la conducción eficiente o eco-driving, ya que presenta grandes ahorros de combustible a nivel individual. Aunque estas reducciones están suficientemente documentadas en la literatura, muy pocos estudios se centran en el efecto que los conductores eficientes pueden tener en el flujo de tráfico, y cuál sería el impacto si se fuera aumentando el porcentaje de este tipo de conductores.

\subsection{Eco-driving. Evolución y definición}

El eco-driving evoluciona a partir del término "hypermiling", consistente en conducir la mayor distancia posible con el mínimo gasto en combustible.

Debido a la preocupación por las emisiones y el cambio climático, el eco-driving adquiere importancia a partir de los 90. Se puede definir eco-driving como el cambio en el modo de conducción con el objetivo de reducir el consumo de combustible. En esta simple definición se incluyen varios conceptos, en función del nivel de decisión (Sivak y Schoettle, 2012):

- Nivel estratégico: Selección del vehículo y mantenimiento.

- Nivel táctico: Selección de ruta y carga del vehículo.

- Nivel operacional: Comportamiento del conductor.

Centrándonos en el nivel operacional, el comportamiento eficiente se basa en acelerar y frenar suavemente, cambiar de marcha a bajas revoluciones, mantener una velocidad constante y anticiparse al tráfico para evitar paradas en la medida de lo posible (Barkenbus, 2010).

Para influir en el comportamiento del conductor se pueden tomar diversas acciones, ya sea a través de campañas de concienciación, autoescuelas, cursos específicos o con sistemas de ayuda a bordo.

Los resultados reflejan una gran variabilidad, desde ahorros del $2 \%$ en consumo de combustible hasta llegar por encima del $30 \%$ en algún caso. Todos estos ahorros son 
individuales, pero muy pocos estudios tienen en cuenta distintos porcentajes de penetración de este comportamiento entre los conductores. En estos estudios, las primeras evidencias apuntan a que un alto porcentaje de conductores eficientes no es positivo en escenarios con grandes volúmenes de tráfico (Kobayashi, Tsubota y Kawashima, 2007; Qian y Chung, 2011; Orfila, 2011).

A continuación se define la metodología basada en la recogida de datos mediante vehículos flotantes en la sección 2. Su posterior uso en la calibración de un modelo de simulación de tráfico queda reflejado en la sección 3. La sección 4 recoge los resultados y este artículo termina con las conclusiones en la sección 5.

\section{METODOLOGÍA}

\subsection{Recogida de datos}

Dentro del marco del proyecto de investigación europeo ICT-Emissions se desarrolló en la ciudad de Madrid una campaña de recogida de datos con el objetivo de obtener los perfiles de velocidad y consumo de varios vehículos afectados por cuatro medidas, en concreto limites variables de velocidad, control de velocidad por tramo, control de crucero y ecodriving. La recogida de datos se basó en vehículos flotantes equipados que recogían velocidad y consumo con una frecuencia de $1 \mathrm{~Hz}$.

La campaña de recogida de datos tuvo lugar en los meses de marzo y abril de 2013. Se usaron 3 vehículos y nueve conductores que recorrieron un total de $12.600 \mathrm{~km}$. por la autopista metropolitana M30 y otras zonas urbanas.

\subsection{Análisis estadístico de los datos}

Una vez filtrados los datos y eliminados los viajes que contenían errores de recepción valores irreales, se procedió al cálculo de 75 variables relacionadas con el perfil de velocidad, como son: velocidad media, aceleración media, número de paradas, porcentaje de tiempo acelerando, etc.

Para reducir el número de variables se realizó un análisis de componentes principales (PCA) (Field, 2013) que agrupó todas estas variables en tres factores. De cada uno de estos factores se ha seleccionado una variable con el criterio de que tenga una carga alta para su factor y muy baja en el resto de factores. Igualmente se ha tenido en cuenta la facilidad de cálculo en el modelo de micro-simulación. Las tres variables seleccionadas son las siguientes:

- Número de paradas.

- Porcentaje de tiempo en el que la aceleración es mayor que $0,1 \mathrm{~m} / \mathrm{s}^{2}$.

- Porcentaje de tiempo en el que la aceleración es mayor que $1,39 \mathrm{~m} / \mathrm{s}^{2}$. 
Con estas variables, se puede plantear un modelo de regresión múltiple donde la variable dependiente es el consumo de combustible y los predictores son las tres variables citadas.

Este modelo de regresión múltiple resulta con un $\mathrm{R}^{2}$ de 0,729 , lo que nos indica que con las variables extraídas del perfil de velocidad podemos explicar un $72,9 \%$ de la varianza del consumo de combustible.

\section{SIMULACIÓN DE ECO-DRIVING}

\subsection{Selección del itinerario a modelizar}

Para simular el comportamiento del conductor eficiente se ha recurrido a modelizar un tramo de los itinerarios recorridos en la campaña de recogida de datos. En concreto, el segmento elegido se trata de una arteria urbana de 2,2 km de longitud, de dos a 4 carriles por sentido según sentido y regulado por semáforos en algunos de sus cruces mientras que otros se resuelven con pasos superiores e inferiores. Este tramo corresponde al recorrido entre la Glorieta López de Hoyos y la Glorieta de Cuatro Caminos en Madrid. La intensidad media diaria (IMD) ronda los 30.000 vehículos en la mayoría de sus tramos con algunos picos de 2.900 vehículos/hora.

\subsection{Simulación del escenario base}

El proceso de simulación se ha llevado a cabo con el software PTV-Vissim v7. Esta herramienta es un programa de simulación de tráfico a nivel microscópico y basado en el comportamiento individual de cada vehículo. Es uno de los programas de microsimulación más usados a nivel mundial tanto en consultoría como en investigación.

La calibración del escenario base se ha realizado siguiendo las indicaciones del Washington State Department of Transportation (WSDOT, 2014) en cuanto a demanda y tiempo de viaje. Los datos reales de tiempo de viaje e intensidades de circulación se han extraídos de los datos de los vehículos flotantes y de una serie de conteos ad hoc.

Para tener en cuenta la aleatoriedad inherente al tráfico, para cada escenario se han simulado 15 repeticiones variando la semilla aleatoria en la herramienta de simulación y cogiendo como valor valido la media de estas 15 repeticiones.

\subsection{Ajuste de parámetros para simular el comportamiento de conductor eficiente}

\subsubsection{Variación real de las variables de referencia al aplicar eco-driving}

Para observar el efecto de la conducción eficiente en el tramo seleccionado, se ha analizado la variación de las variables especificadas en la sección 2.2, es decir, Número de paradas, aceleraciones mayores a $0,1 \mathrm{~m} / \mathrm{s}^{2}$ y aceleraciones mayores a $1,39 \mathrm{~m} / \mathrm{s}^{2}$. La Tabla 1 refleja las variaciones tras aplicar eco-driving en las variables de referencia y en el consumo de combustible. 


\begin{tabular}{|c|c|c|c|c|}
\hline Variable & N_Stops & Acc $>\mathbf{0 . 1}$ & Acc $<1.39$ & Consumption \\
\hline Urban arterial & $-25,2 \%$ & $1,5 \%$ & $-24,6 \%$ & $-7,5 \%$ \\
\hline
\end{tabular}

Tabla 1 - Variaciones en las variables de referencia tras la aplicación de eco-driving

\subsubsection{Proceso de ajuste de parámetros en el modelo de micro-simulación}

En primer lugar, los valores de aceleración y deceleración se han cambiado de acuerdo con los valores encontrados en los viajes reales. En Vissim, estos cambios equivalen a modificar las funciones de aceleración y deceleración, tanto máximas como deseadas. La Tabla 2 refleja los cambios en la aceleración máxima y deseada en Vissim.

\begin{tabular}{|l|c|c|}
\hline Function & Normal & Eco-driving \\
\hline Maximum acceleration & 3,5 & 3,1 \\
\hline Desired acceleration & 3,5 & 2,3 \\
\hline Maximum deceleration & $-6,0$ & $-4,0$ \\
\hline Desired deceleration & $-2,8$ & $-1,9$ \\
\hline
\end{tabular}

Tabla 2 - Variación de los valores de aceleración y desaceleración máxima y deseada en Vissim.

Después de los cambios en estas funciones, el siguiente paso consiste en el ajuste de los parámetros del modelo de seguimiento (car-following model) que controla el movimiento longitudinal en Vissim. La Tabla 3 refleja los cambios en los parámetros del modelo Vissim.

\begin{tabular}{|l|r|r|}
\hline Function & \multicolumn{1}{|c|}{ Normal } & Eco-driving \\
\hline CC0 (Standstill Distance) & 1.50 & 1.50 \\
\hline CC1 (Headway Time) & 0.90 & 1.50 \\
\hline CC2 & 4.00 & 4.00 \\
\hline CC3 & -8.00 & -10.00 \\
\hline CC4 & -0.35 & -0.50 \\
\hline CC5 & 0.35 & 0.50 \\
\hline CC6 & 11.44 & 11.44 \\
\hline CC7 & 0.25 & 0.05 \\
\hline CC8 & 3.50 & 2.90 \\
\hline CC9 & 1.50 & 1.50 \\
\hline Observed vehicles & 2 & 10 \\
\hline
\end{tabular}

Tabla 3 - Valores finales de los parámetros del modelo de seguimiento en Vissim

Este ha sido un proceso de prueba y error hasta conseguir variaciones de la emisiones de $\mathrm{CO}_{2}$ del mismo orden que las del consumo de combustible real, es decir -7,5\% (Tabla 1). Con los valores especificados en la Tabla 3, conseguimos una disminución de $\mathrm{CO}_{2}$ del $5,6 \%$. Se puede considerar valido ya los valores simulados están entre el valor real y el $72,9 \%$ de ese valor, que como habíamos dicho era la varianza que podemos explicar con 
las variables de referencia.

\subsection{Escenarios a simular}

Se han considerado tres escenarios base de acuerdo con demandas de tráfico diferentes: hora punta, hora valle y hora nocturna. La Tabla 4 refleja la carga de tráfico en cada una de ellas:

\begin{tabular}{|l|r|}
\hline & Arteria urbana \\
\hline Capacidad (veh/h) & 2.758 \\
\hline Hora punta (V/C) & 0,89 \\
\hline Hora valle (V/C) & 0,46 \\
\hline Hora nocturna (V/C) & 0,02 \\
\hline
\end{tabular}

Tabla 4 - Relación volumen/capacidad en cada escenario base

Respecto a los porcentajes de penetración de conductores eficientes, se han considerado los siguientes: $0 \%$ (escenario base), 10\%, 25\%, 50\%, 75\% y 100\%. El total de escenarios simulados es 18 . Se ha supuesto que solo los turismos cambian su forma de conducción. Sin embargo el cálculo de emisiones (modelo VERSIT+) se ha realizado para todos los vehículos en la vía, incluyendo furgonetas, camiones y autobuses.

\section{RESULTADOS}

La siguiente figura muestra las velocidades medias recogidas para los distintos escenarios simulados.

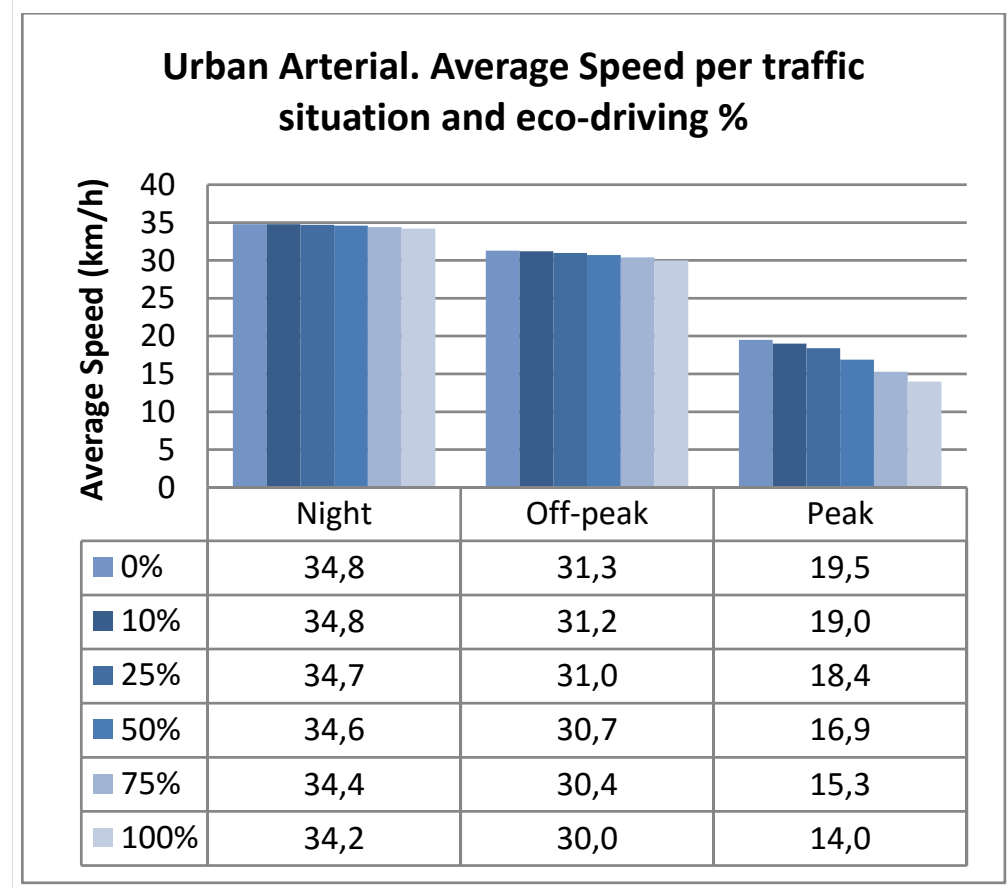

Fig. 1 - Velocidades medias en cada escenario

La figura muestra como la velocidad media decrece cuando aumenta el porcentaje de 
conductores eficiente. Esto se debe principalmente a las aceleraciones más suaves en los semáforos, que disminuyen ligeramente la velocidad y la tasa de descarga. Esto hace que disminuya la tasa de descarga en los semáforos y se ralentice el flujo aún más en horas punta.

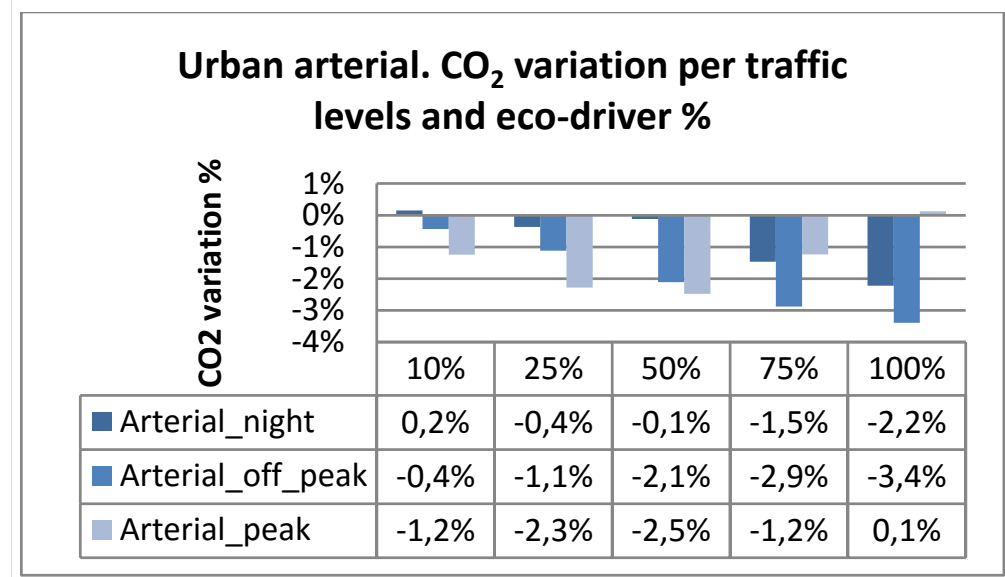

Fig. 2 - Variación de las emisiones de $\mathrm{CO}_{2}$ con respecto a los escenarios base

En relación con las emisiones de $\mathrm{CO}_{2}$, Fig. 2 muestra ahorros en prácticamente todos los escenarios, destacando el $3.4 \%$ en hora valle con el $100 \%$ de conductores eficientes. Las tendencias son similares en el caso de las emisiones de $\mathrm{NO}_{\mathrm{x}}$, donde las emisiones se reducen alrededor del $5 \%$ en hora punta para un $50 \%$ de conductores eficientes.

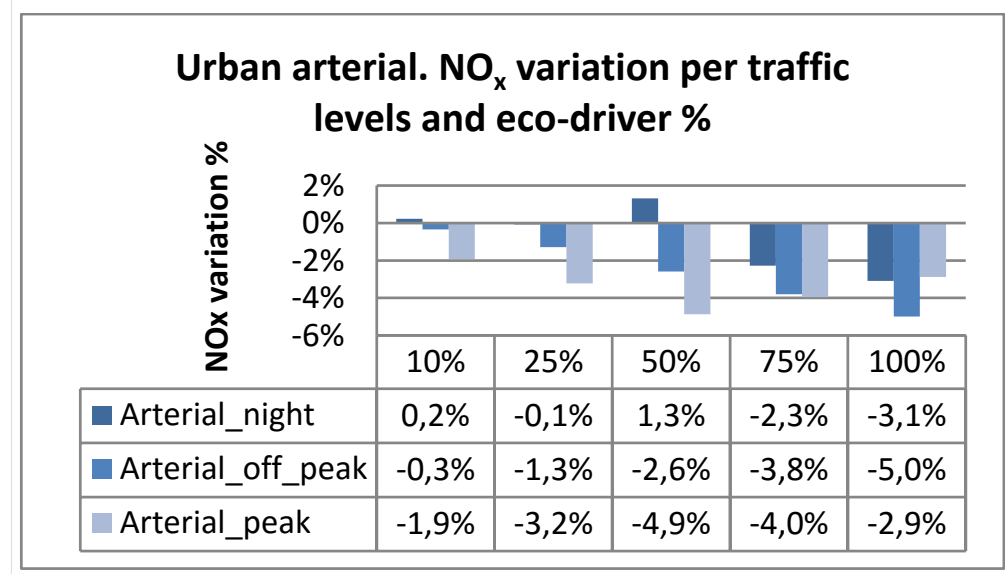

Fig. 3 - Variación de las emisiones de $\mathrm{NO}_{\mathrm{x}}$ con respecto a los escenarios base

\section{CONCLUSIONES}

Este artículo presenta una metodología para simular los efectos de eco-driving en base a datos reales recogidos por vehículos flotantes. Por medio del ajuste de los parámetros del modelo de micro-simulación de tráfico es posible calcular los efectos de diversos porcentajes de conductores eficientes en el flujo de tráfico.

Observando los resultados podemos afirmar que el comportamiento eficiente de los 
conductores afecta al flujo de tráfico. Hemos visto, como según va aumentado el porcentaje de conductores eficientes disminuye la velocidad media, especialmente en condiciones de hora punta. Esta disminución de velocidad provoca un recorte de la capacidad de la vía, aumentando la congestión y repercutiendo negativamente en las emisiones.

También se ha observado que la demanda de tráfico influye claramente en los efectos de eco-driving, tanto en comportamiento del tráfico como en las emisiones. En hora punta los máximos ahorros de emisiones se sitúan en porcentajes de conductores eco cercanos al $50 \%$, mientras que en horas valle, los máximos ahorros se producen con todos los conductores actuando eficientemente.

\section{REFERENCIAS}

BARKENBUS, J. N. (2010). Eco-driving: An overlooked climate change initiative. Energy Policy, 38(2), 762-769.

EUROPEAN ENVIRONMENT AGENCY. (2010). The European Environment. State and Outlook 2010. Urban Environment. Copenhagen, Denmark: Publications Office of the European Union. ISBN 978-92-9213-151-7.

EUROPEAN ENVIRONMENT AGENCY. (2013). Annual European Union greenhouse gas inventory 1990-2011 and inventory report 2013. Copenhagen, Denmark: Publications Office of the European Union.

FIELD, A. (2013). Discovering statistics using IBM SPSS statistics. Sage Publications. KOBAYASHI, I., TSUBOTA, Y., y KAWASHIMA, H. (2007). Eco-driving simulation: Evaluation of eco-driving within a network using traffic simulation. Urban Transport XIII. Urban Transport and the Environment in the 21st Century.

ORFILA, O. (2011). Impact of the penetration rate of ecodriving on fuel consumption and traffic congestion. Paper presented at the YRS11: Young Researchers Seminar 2011, 15p. QIAN, G., y CHUNG, E. (2013). Microscopic simulation study of eco-driving performance at urban intersections. In 13th World Conference on Transport Research, Rio de Janeiro, Brasil.

RAMANATHAN, V., y FENG, Y. (2009). Air pollution, greenhouse gases and climate change: Global and regional perspectives. Atmospheric Environment, 43(1), 37-50.

SIVAK, M., y SCHOETTLE, B. (2012). Eco-driving: Strategic, tactical, and operational decisions of the driver that influence vehicle fuel economy. Transport Policy, 22, 96-99. WASHINGTON STATE DEPARTMENT OF TRANSPORTATION. (2014). Protocol for Vissim Simulation. 